\title{
Ionic Space Charge Driven Organic Photovoltaic Devices
}

\author{
Hadjar Benmansour, Fernando A. Castro, Matthias Nagel, Jakob Heier, Roland Hany, and \\ Frank Nüesch*
}

\begin{abstract}
Most all-organic solar cells rely on undoped electron donor-acceptor heterojunctions. Power-conversion efficiencies depend critically on the photoinduced charge generation at these interfaces such as the charge transport through the layers and collection at the electrodes. Hence, the ability to regulate and control these processes would offer advanced device functionality. Mobile ions are able to create internal electric fields similar to conventional, electronic $p-n$ junctions without having the inconvenience of doping, which often leads to carrier recombination and excited state quenching. We demonstrate that at organic heterointerfaces these ionic junctions can shift the electronic orbital energy level, which allows the direction of electron transfer processes to be controlled. Cationic cyanine dyes offer a convenient model system to study the effect of mobile ions systematically. In conjunction with usually strong electron acceptors such as the Buckminsterfullerene $C_{60}$, and donors such as the poly(p-phenylenevinylene) derivative MEH-PPV, we fabricated bilayer photovoltaic devices to reveal exciting effects due to ionic interfacial space charge. For example, we show that $\mathrm{C}_{60}$ can be turned into an electron donor. Furthermore, oxidative or reductive electron transfer processes can simply be switched on and off with an applied bias, thereby drastically altering device performance and spectral sensitivity.
\end{abstract}

Keywords: Cyanine dye - Organic photovoltaic device

\section{Introduction}

Classical inorganic solar cells based on silicon, gallium arsenide or chalcogenides are based on electronic p-n junctions that are formed between a n-doped and a p-doped semiconductor material. ${ }^{[1]}$ It is the difference between the electronic Fermi levels of the two differently doped materials that gives rise to an electron flow from the ntype to the p-type semiconductor. This charge carrier displacement produces an electric field at the p-n junction. Photon absorption in the classical semiconductors leads promptly to free positive and nega-
${ }^{*}$ Correspondence: Dr. F. Nüesch

Laboratory for Functional Polymers

Swiss Federal Laboratories for Materials Testing

and Research (Empa)

Überlandstrasse 129

$\mathrm{CH}-8600$ Dübendorf

E-Mail: frank.nueesch@empa.ch tive charge carriers that are then spatially separated by the junction. In organic semiconductors, light absorption first leads to a strongly bound electron-hole pair or exciton. ${ }^{[2,3]}$ In heterojunction solar cells made from two complementary organic materials, the exciton diffuses ${ }^{[4,5]}$ until it is split into a more loosely bound electron-hole pair at the interface (Fig. 1). ${ }^{[6]}$ The rather slow interfacial recombination kinetics ${ }^{[7,8]}$ allows the charges to hop away to nearby

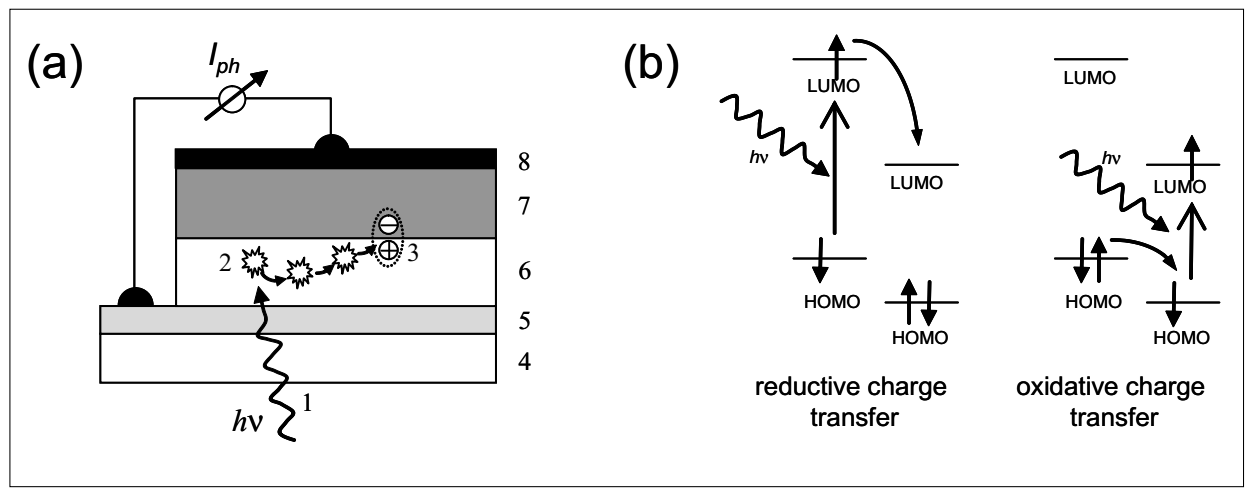

Fig. 1. (a) Working principle of a bilayer organic solar cell composed of a transparent substrate (4), a transparent conductive anode (5), an electron donor (6), an electron acceptor (7), and a cathode (8). Upon light absorption (1), excitons (2) diffuse until they recombine or reach the organic heterointerface where charge generation into free electron and holes takes place (3). (b) Depending on whether the electron donor or electron acceptor is photoexcited, reductive or oxidative charge transfer processes can occur for favourable frontier orbital alignment. sites and eventually to reach the electrodes where photocurrent is collected. ${ }^{[9,10]} \mathrm{A}$ crucial step for efficient photocurrent generation is therefore the heterogeneous electron transfer reaction at the organic interface which, according to Marcus theory, ${ }^{[11]}$ is mainly governed by the relative positions of the lowest unoccupied molecular orbital (LUMO) and highest occupied molecular orbital (HOMO) energy levels of the donor and acceptor materials. High power- 
conversion efficiencies depend critically on the balanced adjustment of the energy levels and their offsets at the heterojunction, to allow both reductive and oxidative charge transfer reactions (Fig. 1). ${ }^{[12]}$

Even though the redox levels of organic conjugated molecules can be conveniently measured in solution, their exact position in the solid state and in particular at a heterogeneous interface is difficult to predict. Impressive potential shifts at electrodes and organic interfaces have been reported. [13-17] Monolayers consisting of dipolar molecules of a few Debye and with a surface density of $10^{18} \mathrm{~m}^{-2}$ easily induce interfacial potential shifts of the order of $1 \mathrm{~V} \cdot{ }^{[18-20]}$ This was used to enhance or impede charge injection into organic materials. Mobile ions have similar effects at interfaces and are a central issue in all electrochemical processes. ${ }^{[21]}$ Potential shifts induced by ions have also been used in optoelectronic devices. Lightemitting electrochemical cells provide Ohmic electrode-polymer contacts when using a polymer blend made of the electroactive conjugated polymer and a polymer electrolyte. Poly(ethylene-oxide) with dissolved lithium triflate proved to readily produce mobile positive and negative ions that accumulate at the electrode surface and facilitate charge injection. ${ }^{[22,23]}$ Heterojunctions built from cationic and anionic molecules show the diffusion of negative and positive counterions across the interface.
Electroluminescence and photovoltaic response in such devices brought evidence that ionic junctions are indeed built by the thermodynamically driven displacement of mobile ionic charges. ${ }^{[24]}$

In this work a cationic cyanine dye with different counterions was used to fabricate heterojunction bilayer photovoltaic devices using a prototypic acceptor $\left(\mathrm{C}_{60}\right)$ and donor (MEH-PPV) material, respectively. We demonstrate that the mobile counterions can be easily displaced by applying an external electric field. It is demonstrated that the performance of photovoltaic devices can be altered tremendously by deploying ionic space charge across the organic interface. The cyanine model compounds allow the rationalization of the conspicuous counterion effect on the spectral response and efficiency of organic bilayer photovoltaic devices.

\section{Experimental Section}

\section{Materials}

Cyanine dye chloride salt $(\mathrm{CyCl})$ and hexafluorophosphate salt (CyPF) were provided by FEW Chemicals (Germany) and used without further purification. Polystyrene sulfonic acid (PEDOT:PSS, Bayer), fullerene $\mathrm{C}_{60}$ (SES Research, 99.95\% purity), tetrafluoropropanol (TFP, Fluka) and chlorobenzene were used as received.

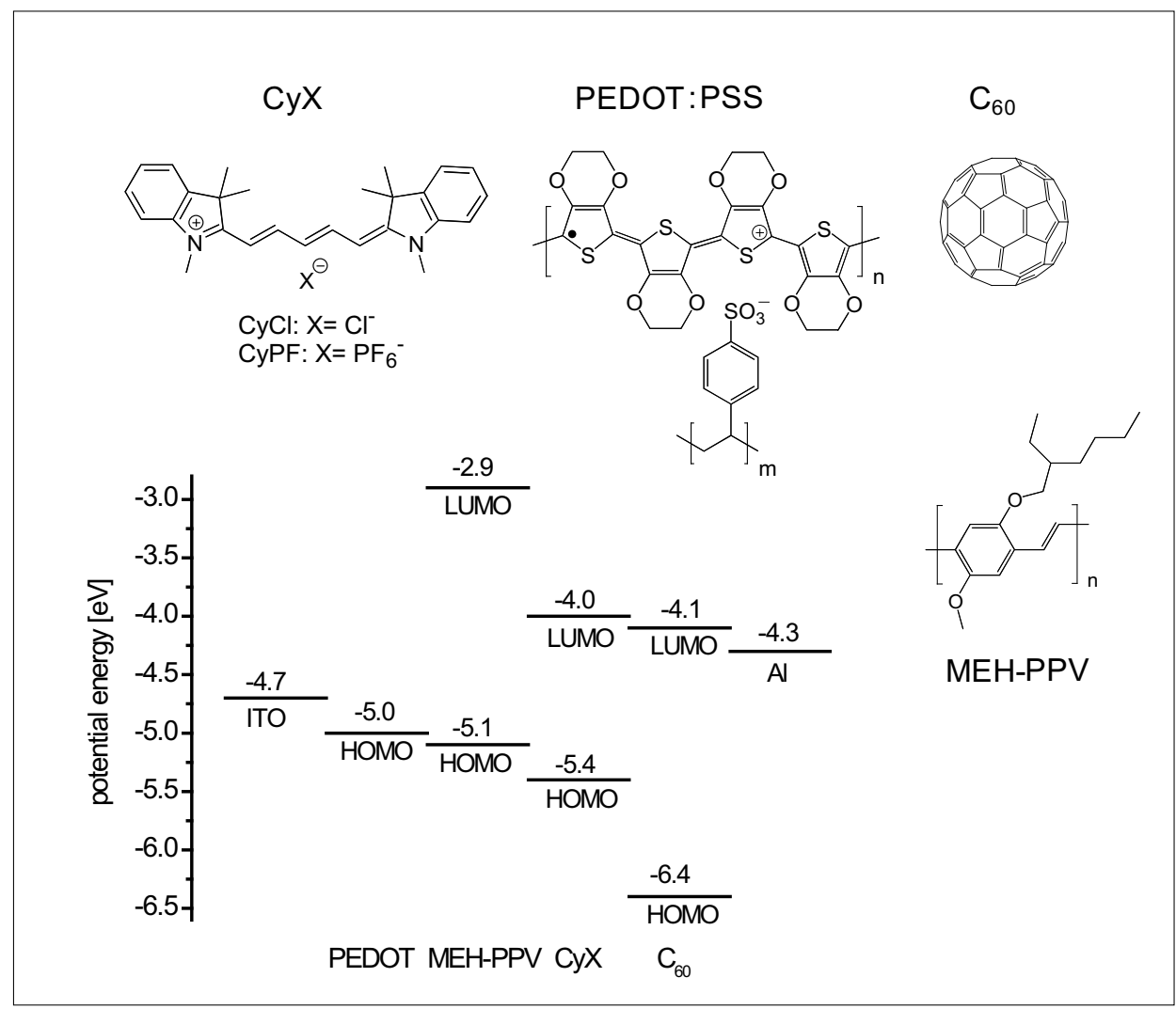

Fig. 2. Chemical structures of the materials used in this work. The HOMO and LUMO frontier energy levels as well as the work functions of the electrodes are indicated.
MEH-PPV (Aldrich, Mn = 40'000-70'000 $\mathrm{g} / \mathrm{mol}$ ) was purified by flash chromatography (THF, silica gel). Chemical structures of the organic compounds used in this work are shown in Fig. 2.

\section{Cyclic Voltammetry}

Cyclic voltammetry measurements were recorded on a PGStat 30 potentiostat (Autolab) using a three electrode cell system with a glassy carbon working electrode, a platinum counter electrode and an $\mathrm{Ag} /$ $\mathrm{AgCl}(0.1 \mathrm{M}$ tetrabutyl ammonium chloride in acetonitrile) reference electrode. Redox potentials of $\mathrm{CyCl}$ and $\mathrm{CyPF}$ were measured in acetonitrile and referenced to the ferrocene/ferrocenium couple. To relate the electrochemical potentials to the corresponding vacuum levels, the potential of $0.69 \mathrm{~V} v s$ NHE was taken for ferrocene and the NHE value of $-4.48 \mathrm{eV} v s$ vacuum was adopted from the literature. ${ }^{[25]}$ Redox potentials of $\mathrm{C}_{60}$ referenced to ferrocene/ferrocenium were taken from the literature.[26] Therefore, the same relationship between the electrochemical scale and the physical scale was applied to obtain the vacuum reduction and oxidation potentials. The highest occupied molecular orbital (HOMO) energy level of PEDOT:PSS was adopted from the literature. ${ }^{[27]}$ Fig. 2 summarizes the lowest unoccupied molecular orbital (LUMO) and HOMO energy levels of the different materials.

\section{Device Fabrication and Characterization}

Solar cell devices were fabricated on patterned indium-tin-oxide coated glasses (ITO, $150 \mathrm{~nm}, 20 \Omega /$ square) that were cleaned in ethanol, acetone and detergent ultrasonic baths. A $90 \mathrm{~nm}$ thick PEDOT:PSS layer was deposited by spin coating and heated in air at $150{ }^{\circ} \mathrm{C}$ for $10 \mathrm{~min}$ to remove residual water. $\mathrm{A} 30 \mathrm{~nm}$ thick $\mathrm{CyCl}$ or $\mathrm{CyPF}$ layer was then spin coated from a $0.5 \mathrm{wt} \%$ solution in TFP on top of PEDOT:PSS, followed by vapour deposition of a $40 \mathrm{~nm}$ thick $\mathrm{C}_{60}$ layer under high vacuum. Finally, a $70 \mathrm{~nm}$ thick aluminium cathode was vapour deposited through a shadow mask, defining four separate solar cells with an active device area of $0.031 \mathrm{~cm}^{2}$. Except for the PEDOT:PSS deposition, the full device fabrication sequence leading to the ITO/ PEDOT:PSS/CyCl or $\mathrm{CyPF} / \mathrm{C}_{60} / \mathrm{Al}$ architecture was carried out under inert atmosphere. Similarly, ITO/PEDOT:PSS/MEH$\mathrm{PPV} / \mathrm{CyPF} / \mathrm{Al}$ devices were fabricated by spin coating a $30 \mathrm{~nm}$ thick MEH-PPV layer from a $0.5 \mathrm{wt} \%$ solution in chlorobenzene on PEDOT:PSS. Then, the film was dried at $50{ }^{\circ} \mathrm{C}$ for $90 \mathrm{~min}$. Subsequently, a $70 \mathrm{~nm}$ thick cyanine layer was deposited from a 1 wt $\%$ solution in a 10:1 TFP-chlorobenzene mixture. Absorbance of the bilayer was measured to ensure that no dissolution of 
the underlying MEH-PPV layer occurred during spin coating of the cyanine.

Thicknesses of the organic films were measured using an Ambios XP-1 profilometer, while morphology analyses were carried out on a Nanosurf Mobile $\mathrm{S}$ atomic force microscope in tapping mode employing rectangular silicon cantilevers (Mikromasch, Nanosensors). Optical absorption spectra were measured with a Varian Cary 50 spectrophotometer. Dynamic secondary mass spectroscopy (D-SIMS) analysis (Physical Electronics 6650) utilized a 3kV, $400 \mathrm{nA} \mathrm{Cs}^{+}$primary ion beam rastered over a surface area of $400 \times 500 \mu \mathrm{m}$. For mass detection only those negative ions emitted form the central point corresponding to $15 \%$ of the crater area were accepted.

Photovoltaic device performance was characterized under nitrogen atmosphere using a monochromator (Oriel 74000) with a xenon lamp light source to obtain the incident photon-to-current conversion efficiency (IPCE) according to IPCE $=1240$ $\times J_{\mathrm{SC}} \times \lambda^{-1} \times P_{\text {in }}^{-1}$, where $J_{\mathrm{SC}}\left(\mathrm{mA} \mathrm{cm}{ }^{-2}\right)$ is the short-circuit photocurrent density and $P_{\text {in }}\left(\mathrm{mW} \mathrm{cm}^{-2}\right)$ is the incident light power at wavelength $\lambda(\mathrm{nm})$.

\section{Results and Discussion}

The family of $\mathrm{Cy}^{+} \mathrm{X}^{-}$cyanine salts allows a systematic investigation of the influence of mobile ionic charge on the generation and flow of electronic current in organic photovoltaic devices. Only the chloride and hexafluorophosphate salts are discussed here as representatives of a small and a large anion, respectively. Both salts showed identical redox levels and absorption spectra in solution. To deploy ions across the organic interface in a controlled way, the bilayer photovoltaic cell architecture is appropriate since an electric field can straightforwardly be applied perpendicularly to the interface (Fig. 1a). The active layers consist of the cyanine film and a complementary photoactive layer of either the excellent electron acceptor $\mathrm{C}_{60}$, or the widely used MEH-PPV conjugated polymer acting as donor in the photoexcited state. A different layer sequence is used for both types of devices, respectively. Since the thin film quality sensitively depends on the counterion, atomic force microscopy measurements were performed to confirm the smoothness and continuity of the cyanine films.

The processes at the heterojunction in the presence of mobile ions can be readily understood. In the as-prepared organic bilayer, mobile ions diffuse from the cyanine film into the non-ionic component because of a concentration gradient. This establishes an electric field across the junction that counteracts the diffusion. Ions will equili- brate at the interface when the fluxes due to diffusion and drift are balanced. This represents the situation for the relaxed, electrically non-contacted device (flat band condition). For the measurement of the short-circuit current, the electrical connection between anode and cathode creates an extra built-in field that will act on the ions. By monitoring the photocurrent change under short-circuit conditions as function of time, we determined a typical time scale of more than $100 \mathrm{~min}$ for the redistribution of $\mathrm{PF}_{6}{ }^{-}$ions under this extra field due to the different electrode work functions, while the redistribution of $\mathrm{Cl}^{-}$was much faster and typically completed after 10 min. The time for the spectral photocurrent measurement is approximately $2 \mathrm{~min}$; therefore, for both ions we are able to map the initial relaxed state of the device. We also applied strong external electric fields to drive a more important concentration of ions across the heterointerface, and the time to restore the initial state in this case took several days. Therefore, also then we are able to map ion displacement just after biasing by performing a spectral photocurrent measurement under short-circuit conditions.

First, we discuss ITO/PEDOT:PSS/ $\mathrm{CyPF} / \mathrm{C}_{60} / \mathrm{Al}$ bilayer devices. It is not $a$ pri- ori clear whether $\mathrm{CyPF}$ will act as electron donor since the energy difference between the LUMO of $\mathrm{CyPF}$ and $\mathrm{C}_{60}$ is, according to cyclic voltammetry measurements, rather small (Fig. 2). Distributing mobile ions across the cyanine- $\mathrm{C}_{60}$ interface may change the energy levels and their offsets at the heterojunction and alter the donor and acceptor properties.

If a negative bias was applied at the ITO anode (Fig. 3a), the electric field developed perpendicularly to the organic layers exerted a force on the $\mathrm{PF}_{6}{ }^{-}$ions pushing them into the $\mathrm{C}_{60}$ layer. This decreased the spectral photocurrent quantum yield but without changing the shape of the spectrum (Fig. 3a) that is characterized by the $\mathrm{C}_{60}$ and cyanine contributions at $450 \mathrm{~nm}$ and $700 \mathrm{~nm}$, respectively. This means that both the reductive and oxidative electron transfer processes are still possible after $\mathrm{PF}_{6}^{-}$ion displacement. The observed current decrease can be attributed to the space charge built up in the exciton dissociation zone of the device that is opposite to the usual charge transport direction, driving electrons to the aluminium cathode and holes to the anode. As a result, recombination between holes and electrons became more important and reduced the photocurrent. Allowing for some relaxation time partly restored the initial photocurrent,

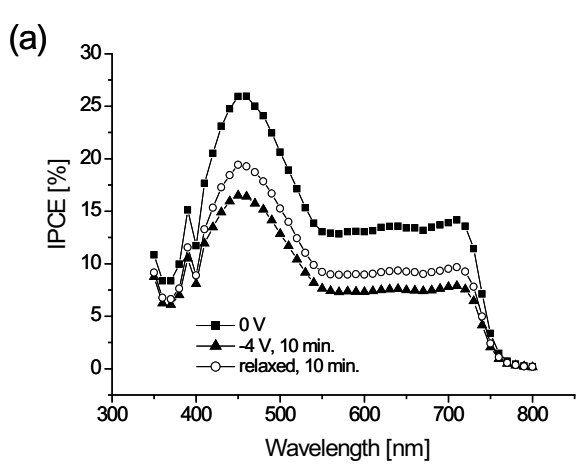

(b)

(c)

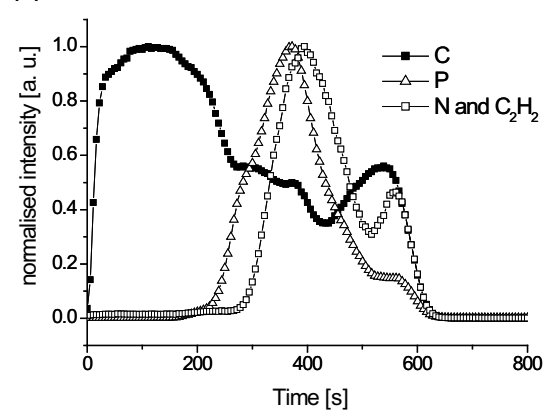

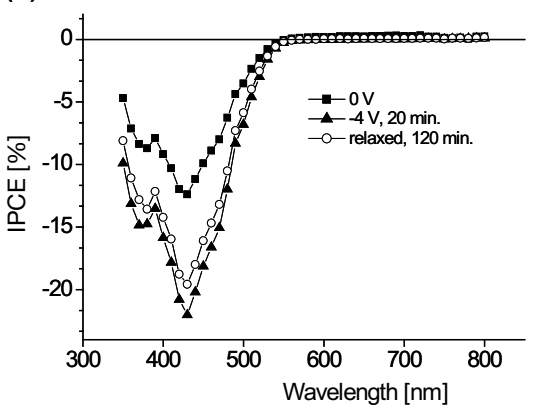

(d)

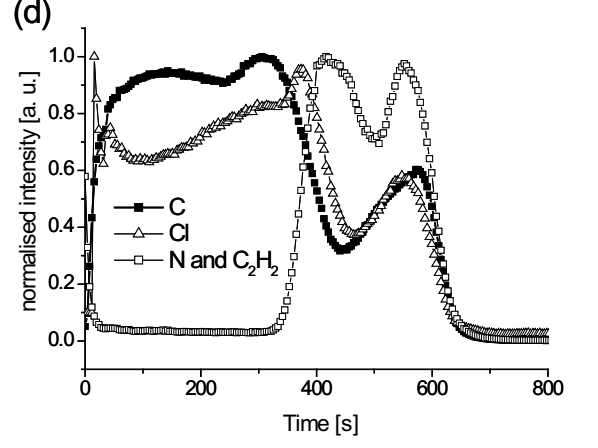

Fig. 3. Photocurrent action spectra (IPCE) of (a) ITO/PEDOT:PSS/CyPF/C 60 /Al devices and (b) ITO/ PEDOT:PSS/CyCl/ $\mathrm{C}_{60} / \mathrm{Al}$ devices in the relaxed state at $0 \mathrm{~V}$ biasing and as a function of biasing time at $-4 \mathrm{~V}$. The IPCE characteristics recorded after a certain relaxation time after biasing are also indicated. Dynamic secondary ion mass spectra obtained by using a $\mathrm{Cs}^{+}$primary ion beam impinging on the $\mathrm{C}_{60}$ surface area of (c) ITO/PEDOT:PSS/CyPF/C 60 and (d) ITO/PEDOT:PSS/CyCl/C $60^{*}$ The chemical structure corresponding to the detected masses is indicated in the graph. In the D-SIMS profiles, the cyanine film can be identified by the mass signal corresponding to nitrogen, on which the mass of $\mathrm{C}_{2} \mathrm{H}_{2}$ is superimposed. The carbon signal measured at small ion bombardment times corresponds to $\mathrm{C}_{60}$. 
as indicated in Fig. 3a. At longer relaxation times, the original performance was re-established, demonstrating that the effect is reversible and induced by mobile ions. The starting situation could also be restored by applying an opposite bias.

A very different situation occurred in the case of the chloride salt. Even at zero applied bias, the photocurrent had the inversed sign in the $\mathrm{C}_{60}$ region (indicated by the negative quantum efficiency in Fig. $3 b)$ and no photocurrent contribution after photoexcitation of the cyanine was observed. By applying a negative bias this anomalous effect was even enhanced. Obviously, chloride anions are more mobile than $\mathrm{PF}_{6}{ }^{-}$ions and build up an important space charge across the heterojunction. As we inferred from dynamic SIMS measurements performed on unbiased organic bilayers, chloride ions diffuse massively into the $\mathrm{C}_{60}$ layer, while $\mathrm{PF}_{6}{ }^{-}$anions stay closer to the interface (Figs $3 \mathrm{c}$,d). The interfacial charge density in the case of chloride is so important that the LUMO energy level of $\mathrm{C}_{60}$ is raised above the LUMO level of the cyanine. As a consequence, $\mathrm{C}_{60}$ becomes the electron donor while the cyanine takes over the role of the acceptor (see the simplified scheme in Fig. 4a). The HOMO level of $\mathrm{C}_{60}$ comes closer to that of the cyanine but still is too low to permit oxidative charge transfer to the cyanine (Fig. 4b). Therefore, no electron transfer reactions take place after photoexcitation of the cyanine at wavelengths above 600 nm (Fig. 3b). These two examples demonstrate the drastic effect of counterion displacement on the photovoltaic properties. Mobile ions can build up very substantial ionic space charge that can even invert the direction of the photocurrent.

As a second example we used the cyanine with a conjugated polymer layer. The LUMO energy difference between MEHPPV and CyPF in solution is very large $(>1$ eV, Fig. 2). This suggests that in the ITO/ PEDOT:PSS/MEH-PPV/CyPF/Al device architecture reductive electron transfer after MEH-PPV photoexcitation will occur in any case. The photocurrent spectrum of the relaxed devices showed indeed the expected MEH-PPV contribution at about $520 \mathrm{~nm}(0$ V, Fig. 5a). In addition, strong photoluminescence quenching of MEH-PPV-cyanine films revealed that a low volume fraction of isolated cyanine molecules diffuse into the conjugated polymer film during solution deposition. Consequently, a large part of initially produced electrons and holes will not find percolating conduction paths to the heterojunction and have to decay by recombination. This explains why the current collection efficiency after MEH-PPV excitation is rather small $(<1 \%)$.

In the relaxed device, the tiny cyanine contribution at around $700 \mathrm{~nm}$ indicates

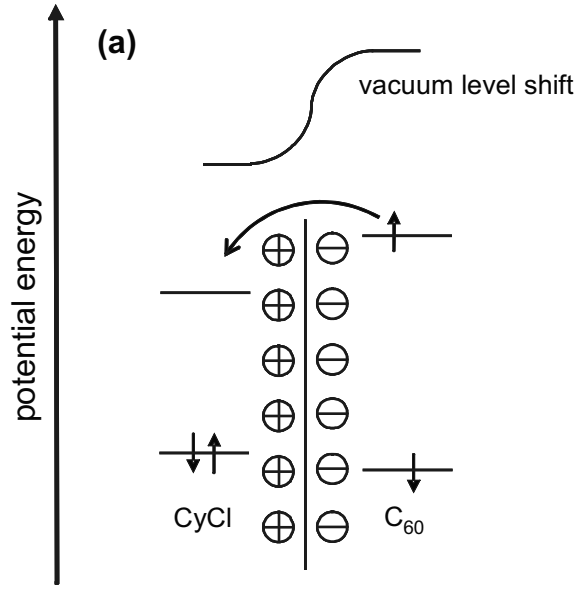

Inverted reductive photocurrent generation (b)

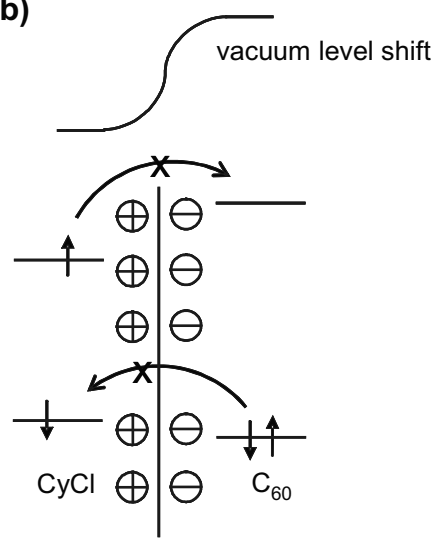

forbidden reductive and oxidative photocurrent generation

Fig. 4. Potential energy diagram in the case of ITO/PEDOT:PSS/CyCI/ $\mathrm{C}_{60} / \mathrm{Al}$ devices. (a) Due to the strong internal field created by the displacement of chloride anions across the organic heterointerface, the photocurrent generated upon excitation of $\mathrm{C}_{60}$ is inverted and $\mathrm{C}_{60}$ now plays the role of the electron donor. (b) Upon cyanine excitation neither reductive nor oxidative photocurrent can be produced. Cyanine cations are indicated with a plus sign and $\mathrm{Cl}^{-}$counter anions by a minus sign.

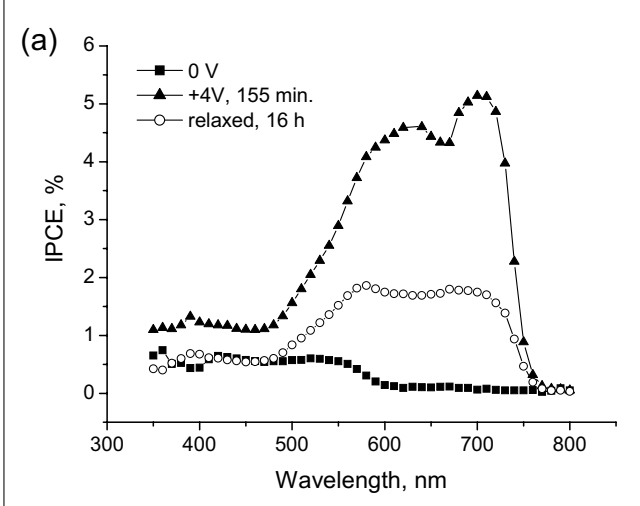

(b)

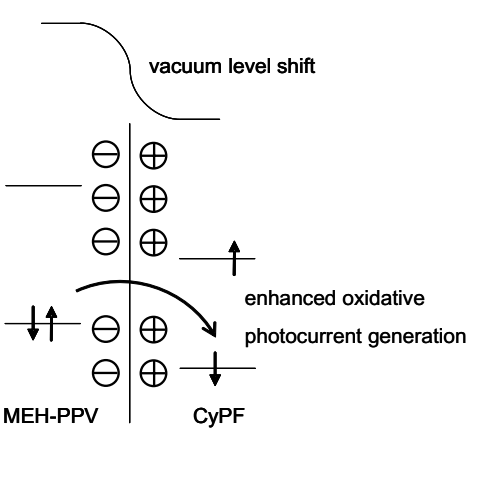

Fig. 5. (a) Photocurrent action spectrum (IPCE) of the ITO/PEDOT:PSS/MEH-PPV/CyPF/AI relaxed device and after biasing at $+4 \mathrm{~V}$. The IPCE characteristic after a long relaxation time following the biasing is also indicated. (b) Potential energy diagram after applying a positive bias to the ITO electrode. Cyanine cations are indicated with a plus sign and $\mathrm{PF}_{6}{ }^{-}$counter anions by a minus sign.

that oxidative electron transfer is not efficient. The two HOMO energy levels are very close (Fig. 2) making diffusion of photogenerated holes into the cyanine layer possible, thereby reducing the photocurrent. A completely different situation was obtained when a positive bias was applied to the device. A huge increase in the photocurrent is observed together with a drastic spectral change. Under the applied electric field, $\mathrm{PF}_{6}{ }^{-}$ions move into the MEH-PPV layer where they build up an interfacial charge that is so important that the $\mathrm{HO}$ MO energy level of MEH-PPV is raised well above that of the cyanine (Fig. 5b). Oxidative electron transfer then becomes efficient. Again, the system is reversible and the ionic junction gradually relaxes when the bias is turned off. To reach the initial photocurrent, however, several days of relaxation are required. This second example demonstrates the ability to modify the spectral sensitivity of a solar cell by displacing ions across the organic heterointerface.

\section{Conclusions}

We have demonstrated the tremendous impact of mobile ions on the photovoltaic properties of organic thin film devices. Ionic space charge can be arranged at organic heterojunctions to tune the microscopic processes such as photoinduced electron transfer on which photovoltaic action relies. Thereby, ionic charge can be used to control the flow of electronic current. For example, the observation that $\mathrm{C}_{60}$ can be converted into an electron donor is note- 
worthy, since normally this compound is one of the best electron acceptors used in organic solar cells. In addition, the spectral sensitivity can be changed and the device simply be turned on and off at a given wavelength. Such features might pave the way to organic solid-state devices with new functionalities. In future investigations, we will design ionic space charge to enhance charge transport in the organic layers, which is often a bottleneck to increased organic solar cell efficiency.

\section{Acknowledgements}

We thank Tom Mates (MRL, UCSB) for performing D-SIMS measurements. We acknowledge funding from COST (Coopération européenne dans le domaine de la recherche scientifique et technique).

Received: October 12, 2007

[1] S. M. Sze, 'Physics of Semiconductor Devices', 2nd ed. John Wiley \& Sons, New York, 1981, p 790.

[2] M. Knupfer, Appl. Phys. A: Mater. Sci. Proc. 2003, 77, 623.

[3] I. G. Hill, A. Kahn, Z. G. Soos, R. A Pascal, Chem. Phys. Lett. 2000, 327, 181.

[4] T. Stubinger, W. Brutting, J. Appl. Phys. 2001, 90, 3632.

[5] J. J. M. Halls, K. Pichler, R. H. Friend, S. C. Moratti, A. B. Holmes, Appl. Phys. Lett. 1996, 68, 3120.

[6] C. J. Brabec, G. Zerza, G. Cerullo, S. De Silvestri, S. Luzzati, J. C. Hummelen, S. Sariciftci, Chem. Phys. Lett. 2001, 340, 232.

[7] A. F. Nogueira, I. Montanari, J. Nelson, J. R. Durrant, C. Winder, N. S. Sariciftci, J. Phys. Chem. B 2003, 107, 1567.

[8] T. Offermans, S. C. J. Meskers, R. A. J. Janssen, J. Chem. Phys. 2003, 119, 10924.

[9] B. P. Rand, D. P. Burk, S. R. Forrest, Phys. Rev. B 2007, 75, 115327.
[10] P. W. M. Blom, V. D. Mihailetchi, L. J. A Koster, D. E. Markov, Ad. Mater. 2007, 19, 1551.

[11] R. A. Marcus, N. Sutin, Biochim. Biophys. Acta 1985, 811, 265.

[12] C. W. Tang, Appl. Phys. Lett. 1986, 48, 183.

[13] R. Schlaf, C. D. Merritt, L. A. Crisafulli, Z. H. Kafafi, J. Appl. Phys. 1999, 86, 5678.

[14] G. Heimel, L. Romaner, J. L. Bredas, E. Zojer, Phys. Rev. Lett. 2006, 96, 196806.

[15] S. Braun, W. R. Salaneck, Chem. Phys Lett. 2007, 438, 259.

[16] R. Schlaf, B. A. Parkinson, P. A. Lee, K. W. Nebesny, N. R. Armstrong, J. Phys. Chem. B 1999, 103, 2984.

[17] E. W. Forsythe, V. E. Choong, T. Q. Le, Y. L. Gao, J. Vacuum Sci. Technol. A: Vacuum Surf. Films 1999, 17, 3429.

[18] F. Nuesch, M. Carrara, L. Zuppiroli, Langmuir 2003, 19, 4871.

[19] F. Nuesch, F. Rotzinger, L. Si-Ahmed, L. Zuppiroli, Chem. Phys. Lett. 1998, 288, 861.

[20] I. H. Campbell, S. Rubin, T. A. Zawodzinski, J. D. Kress, R. L. Martin, D. L. Smith, N. N. Barashkov, J. P. Ferraris, Phys. Rev. B 1996, 54, 14321.

[21] A. J. Bard, 'Electrochemical Methods: Fundamentals and Applications', Wiley, New York, 2001.

[22] J. C. deMello, N. Tessler, S. C. Graham, R. H. Friend, Phys. Rev. B 1998, 57, 12951.

[23] Q. B. Pei, G. Yu, C. Zhang, Y. Yang, A. J. Heeger, Science 1995, 269, 1086.

[24] D. A. Bernards, S. Flores-Torres, H. D. Abruna, G. G. Malliaras, Science 2006, $313,1416$.

[25] C. P. Kelly, C. J. Cramer, D. G. Truhlar, J. Phys. Chem. B 2007, 111, 408.

[26] T. Suzuki, Y. Maruyama, T. Akasaka, W. Ando, K. Kobayashi, S. Nagase, J. Am. Chem. Soc. 1994, 116, 1359.

[27] N. Koch, A. Kahn, J. Ghijsen, J. J. Pireaux, J. Schwartz, R. L. Johnson, A. Elschner, Appl. Phys. Lett. 2003, 82, 70. 